\title{
Drug-eluting stent pathology—should we still be cautious?
}

\author{
Gaku Nakazawa, Aloke V Finn and Renu Virmani
}

The current FDA-approved drug-eluting stents (DESs; CYPHER ${ }^{\circledR}$ [Cordis, Miami Lakes, FL] and TAXUS $^{\circledR}$ [Boston Scientific, Natick, MA]) have been associated with late stent thrombosis (LST; defined as thrombosis occurring $>30$ days following implantation). The mechanisms predictive of LST are, however, poorly understood. While CYPHER ${ }^{\circledR}$ and TAXUS ${ }^{\circledR}$ stents use different drug coatings, both effectively reduce restenosis.

Pathologic studies are the best methods for detailed analysis of morphologic changes that occur following placement of DESs. We initially reported that both CYPHER ${ }^{\circledR}$ and TAXUS ${ }^{\circledR}$ stents produce delayed arterial healing evidenced by incomplete endothelialization and persistent fibrin deposition when compared with baremetal stents (BMSs) of similar implant duration. Several pathologic risk factors were identified, and later we reported that lack of endothelial strut coverage was the best single correlate of thrombosis. We also observed heterogeneity of healing in the stents with late thrombus.

Underlying lesion anatomy and plaque morphology are important but frequently overlooked factors that can influence healing following DES implantation. In our own pathologic series, the incidence of stent thrombosis in bifurcation DES was higher than that for BMS for implants older than 30 days. These lesions also result in greater delayed arterial healing probably related to the flow disturbance at these locations. Healing is also affected by underlying plaque morphology, such as thin-cap fibroatheroma or ruptured plaques, which frequently underlie acute myocardial infarction (AMI). Stent strut penetration of the necrotic core is commonly observed at autopsy. Our pathologic studies have revealed a significantly higher incidence of LST in patients with AMI than in patients with stable plaque following DES implantation. We have also observed greater and more-uniform arterial healing in patients with stable lesions (i.e. fibroatheroma with or without calcification) than in AMI patients, emphasizing the importance of

\section{Clinicians \\ must, therefore, concentrate on minimizing risk, which includes being aware of the anatomic features associated with greater risk of LST.}

GNakazawa is a Cardiovascular Fellow at the CVPath Institute, Gaithersburg, MD, and AV Finn is Assistant Professor in the Division of Cardiology at the Emory University School of Medicine in Atlanta, GA, USA.

$R$ Virmani is an Advisory Board member of Nature Clinical Practice Cardiovascular Medicine.

\section{Competing interests}

$R$ Virmani declared associations with Abbott Vascular, Alchimedics, Biosensors International, Biotronik, Conor

Medsystems, Cordis Corporation, Medtronic AVE, OrbusNeich, Prescient Medical and Terumo Corporation. See the article online for full details of the relationships. The other authors declared no competing interests.

www.nature.com/clinicalpractice doi:10.1038/ncpcardio1093 patient selection. Although two pivotal studies showed similar rates of stent thrombosis in AMI patients treated with DESs versus BMSs, the limited duration of follow-up (i.e. 1 year) precludes any definitive conclusion about the safety of this practice as most of these patients are receiving long-term antiplatelet therapy. A more recent clinical study has demonstrated that $\mathrm{AMI}$ is an independent risk factor for LST and associated with raised mortality risk.

Given these findings, bifurcation and AMI stenting should be avoided to minimize the risk of LST with DESs. As the time course of complete healing with DES is unknown, the optimum duration of antiplatelet treatment remains to be determined. Even so, some cases of very LST (i.e. beyond 1 year) have been reported despite antiplatelet therapy, emphasizing the complexity of this issue. Clinicians must, therefore, concentrate on minimizing risk, which includes being aware of the anatomic features associated with greater risk of LST.

Another complication reported in patients with DES is a localized hypersensitivity reaction in the underlying coronary artery. Our observations indicate that this phenomenon is limited to the use of the CYPHER ${ }^{\circledR}$ stent. Conversely, extensive fibrin deposition with or without stent malapposition is a more frequent finding with the TAXUS $^{\circledR}$ stent. Hypersensitivity to CYPHER ${ }^{\circledR}$ stents invariably results in LST, whereas excessive fibrin deposition may or may not be associated with LST. The specific drugs and polymers used are likely to be responsible for these adverse reactions.

DESs are a major innovation in interventional cardiology. We have no doubt that DESs are an effective treatment for the prevention of restenosis and can be safely used when patients are properly selected. There is, however, room for improvement. Thus, with the next generation of DESs, greater attention will be given to strut thickness, polymer choice, drug dose, and release kinetics - all of which are currently being pursued vigorously. 